\title{
How can Licel help the ATMOHEAD community?
}

\author{
Bernd Mielke ${ }^{\mathrm{a}}$ \\ Licel GmbH, Gustav-Meyer-Allee 25, 13355 Berlin, Germany
}

Abstract. Licel is a long term contributor of instruments for Lidar research. We show how well our products match the Lidar requirements.

\section{Introduction}

Licel is a privately owned company focusing on Lidar equipment since 1994. We supply instruments to several participants of the ATMOHEAD community such as

- Pierre Auger Observatory - Lubljana [1],

- CEILAP Argentina [2],

- UAB Barcelona [3],

- LUPM Montpellier [3],

- University Napoli/Catania [4].

All Licel detector and data acquisition systems are designed for high linearity in wide dynamic range applications. We address the special needs of the Lidar community such as multi-wavelength systems, low trigger jitter, high laser repetition rates and additional housekeeping data integration.

\section{Transient recorder}

Lidar signals can be acquired with our standard transient recorder combining a 12 bit analog acquisition with a $250 \mathrm{MHz}$ photon counter. Both signals can be combined to a single signal with up to 5.5 orders of magnitude, when used with a suitable PMT [5-7].

\subsection{6 bit transient recorder}

Tropospheric Lidars might require higher dynamic range. Here the new 16 bit transient recorder should be used. It has a higher analog SNR, a pretrigger and can accumulate up to $64 \mathrm{k}$ shots.

\subsection{High repetition rate transient recorder}

The HR type transient recorder has $200 \mathrm{~ns}$ wide bins and an acquisition duty cycle of 1 . It will work perfectly in combination with high repetition rate lasers. It has a presettable number of bins and averages $64 \mathrm{k}$ shots.

a e-mail: mielke@licel.com

\section{Detectors UV, visible}

Licel provides photomultiplier modules matching the transient recorder.

The modules use a stabilized voltage divider to deal with the dynamic range requirements in a Lidar. If the near field signal blinds the detector the photomultiplier modules can be additionally equipped with a gating circuit.

Bialkali and multialkali cathode materials as well as a fused silica window are available to cover the spectral region from $160 \mathrm{~nm}$ to $850 \mathrm{~nm}$.

\section{Detectors NIR, IR}

For lasers emitting wavelengths of $1064 \mathrm{~nm}$ we provide the $\mathrm{Si}-\mathrm{APD}$ with a 1 or $3 \mathrm{~mm}$ diameter detector size. The APD comes with an aspheric lens, an xyz translation stage and a power supply. The only optical requirement is a parallel incoming beam.

For lasers emitting in the range of $1100 \mathrm{~nm}-1650 \mathrm{~nm}$ the InGaAs-APD with a $0.5 \mathrm{~mm}$ detector diameter will detect the signal. It also comes with a lens, a xyz stage and a power supply. Due to the smaller detector size the requirements for the optical layout are greater.

\section{Synchronization}

To synchronize the laser with your experiment a small trigger generator module can be used. The following 4 outputs are supplied:

- Laser Lamp,

- Laser Q Switch,

- Acquisition,

- Gating.

\section{Laser power}

The Laser Monitor module will record the signal either from a photodiode or a pyroelectric power meter head. Laser power fluctuation can be detected by recording the laser pulse energy values for each laser shot. The reading software works together with the Aquis software.

This is an Open Access article distributed under the terms of the Creative Commons Attribution License 4.0, which permits unrestricted use, distribution, and reproduction in any medium, provided the original work is properly cited. 


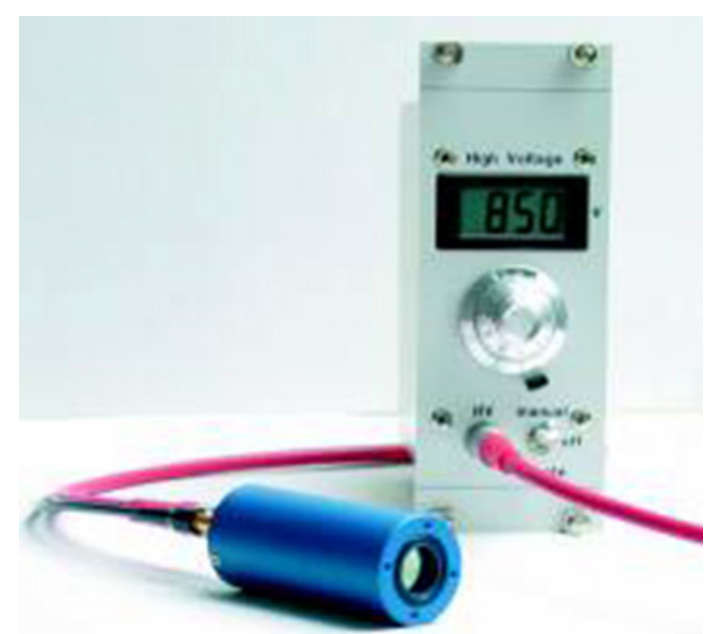

Figure 1. PMT Module.

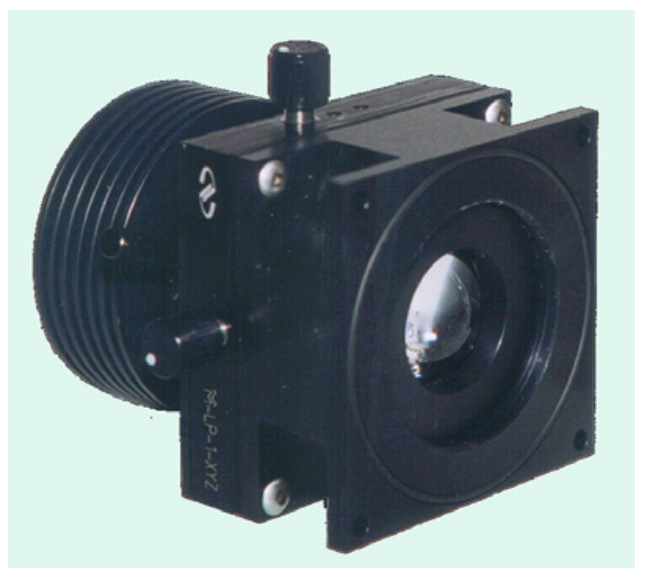

Figure 2. APD Module.

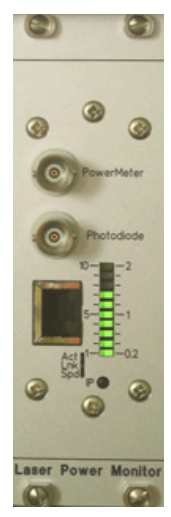

Figure 3. Laser Monitor.

\section{Alignment}

For systems where the laser beam needs to be actively kept in the field of view we supply a bore sight module [8].

It will count single photon pulses in

- 4 signal quadrants,

- 4 background quadrants,

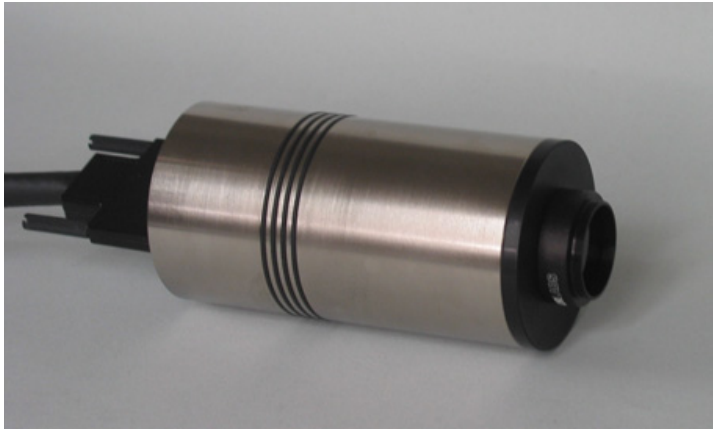

Figure 4. Bore sight detector.

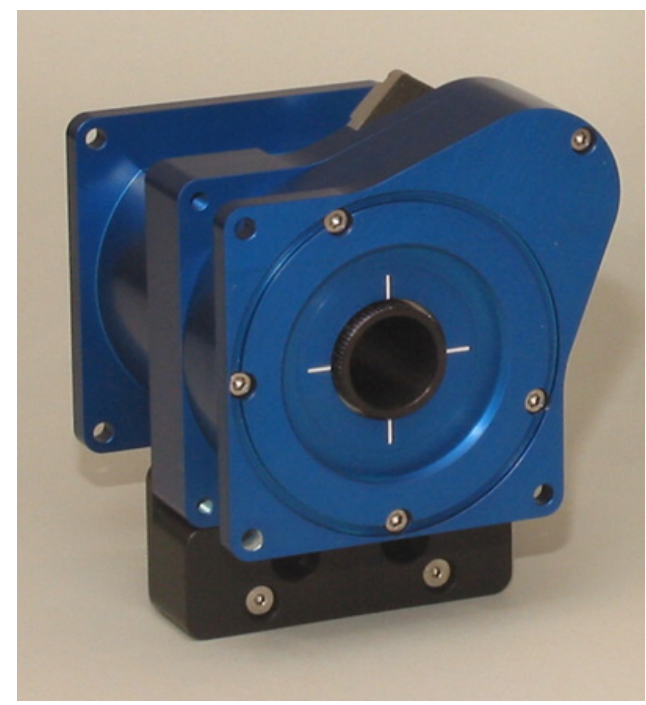

Figure 5. Polarotor.

- and produce a the steering signal, which is used to actively keep the alignment in 2 dimensions.

- The module requires minimal driver adaption for existing drives of $\mathrm{X}-\mathrm{Y}$ stages.

\section{Polarization}

For the aerosol depolarization measurements the polarotor adds (de-)polarization measurements to the multispectral detection systems [9].

- A rotating Glan Thompson prism is used to separate p- and s-polarized signal contributions.

- The integrated trigger generator synchronizes the laser flash-lamp and Q-switch pulses.

\section{Multispectral Lidar Detector}

The 32 channel multispectral lidar detector allows simultaneous detection of 32 wavelengths in parallel.

- Aerosol characterization - Biomass burning detection - Fluorescence Lidar [10,11].

- Liquid water - Water vapor separation - Water vapor Raman [12]. 


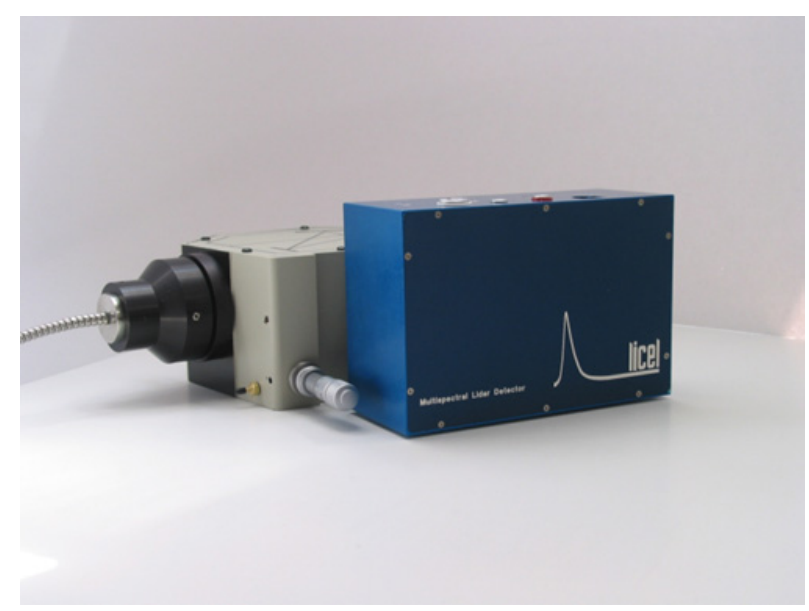

Figure 6. Multispectral Lidar Detector.

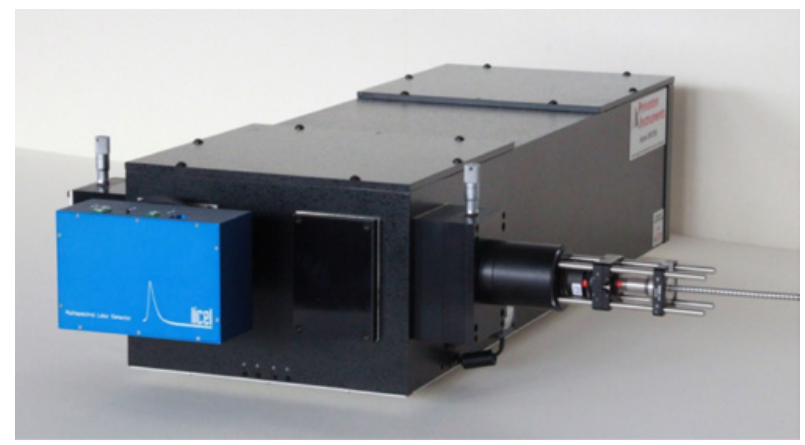

Figure 7. Multispectral Lidar Detector with customer monochromator.

The system starts with

- a fiber bundle transferring a circular beam into a rectangular shape

- the monochromator images the multiple wavelengths on the cathodes of a multianode PMT

- 32 counters deliver 32 photon counting traces

- via Ethernet the traces are transferred to a PC.
The system can be adapted to customers existing monochromators as long as they have at least a $25 \mathrm{~mm}$ wide image field size.

\section{References}

[1] Rizi, V., Tonachini, A., Iarlori, M., Visconti, G., The European Physical Journal Plus, 127, 92 (2012)

[2] Wolfram, E., Salvador, J., D’Elia, R., Casiccia, C., Paes Leme, N., Pazmino, A., Porteneuve, J., GodinBeekman, S., Nakane, H., Quel, E., Journal of Optics A: Pure and Applied Optics, 10, 104021 (2008)

[3] Barcelo, M., et al., "Development of Raman LIDARs made with former CLUE telescopes for CTA", Proceedings of the 32nd ICRC, Bejing, 2011

[4] Pisani, G., Boselli, A., Coltelli, M., Leto, G., Pica, G., Scollo, S., Spinelli, N., Wang, X., Atmospheric Environment, 62, 34-40 (2012)

[5] Newsome, R., Turner, D., Mielke, B., Clayton, M., Ferrare, R., Sivaraman, C., Applied Optics, 48, 3903-3914 (2009)

[6] Whiteman, D., Rush, K., Rabenhorst, S., Welch, W., Cadirola, M., McIntire, G., Russo F., et al., J. Atmos. Oceanic Technol., 27, 1781-1801 (2010)

[7] Zhang Y., Yi, F., Kong, W., Yi, Y., Applied Optics, 53, 7312-7320 (2014)

[8] Mishra, S., Turner, D.D., Newsom, R.K., Evans, S.M., Goldsmith, J., AGU Fall Meeting Abstracts (Vol. 1, p. 02) (2012)

[9] Nott, G., Duck, T., Doyle, J., Coffin, M., Perro, C., Thackray, C., Drummond, J., McCullough E., Sica, R., Journal of Atmospheric and Oceanic Technology, 29, 221-234 (2012)

[10] Immler, F., Engelbart, D., Schrems, 0., Atmos. Chem. Phys., 5, 345-355 (2005)

[11] Sugimoto, N., Huang, Z., Nishizawa, T., Matsui, I., Tatarov, B., Optics Express, 20, 20800-20807 (2012)

[12] Jens, Reichardt, J. Atmos. Oceanic Technol, 31, 1946-1963 (2014) 